\title{
Impact on Bones and Joint during Pregnancy and Breastfeeding: A Short Note
}

\author{
Maria A Grácio* \\ Institute of Hygiene and Tropical Medicine / New University of Lisbon, Lisbon, Portugal
}

${ }^{\star}$ Corresponding author: Maria A Grácio, Institute of Hygiene and Tropical Medicine / New University of Lisbon, Rua da Junqueira 100, 1348-008 Lisbon, Portugal; Email: mameliahelm@ihmt.unl.pt

Received: July 01, 2019; Accepted: July 11, 2019; Published: July 20, 2019;

\section{Short Commentary}

Bone is the name given to hard extremely dense connective tissue that forms the human skeleton. Individual bones may be classed as long, short, flat or irregular. Bones not only form the skeleton but also act as stores for mineral salts and play an important part in the formation of blood cells. Haematopoiesis is the process of production of blood cells and platelets which continues throughout life, replacing aged cells (which are removed from the circulation). In healthy adults, haematopoiesis is confined to the bone marrow, but in embryonic life and in early infancy, as well as in certain diseases, it may occur in other sites (extramedullary haematopoiesis). Joint is the point when two or more bones are connected. The opposing surfaces of bone are lined with cartilaginous fibrous or soft (synovial) tissue. The three main classes of joint are diarthrosis (freely movable), amphiarthrosis (slightly movable) and synarthrosis (unmovable). Between the causes that can damage the bones and joints are: (i) pregnancy; and (ii) breastfeeding. Here, our objective is: 1- summarized (presents) those causes and their effects based on publications that we consider relevant, for divulgation of the impact on bones and joints during pregnancy and breastfeeding, for physicians and other health personnel to bear in mind this when assisting mothers of babies and infants.

In [1] the authors have" identified 35 women who have developed osteoporosis during or shortly after pregnancy and in only six of them could a recognized underlying cause be suggested. These findings would suggest that idiopathic osteoporosis associated with pregnancy may be more common than the current literature suggests".

In [2] the authors suggest "that calcium needed for fetal skeletal growth during pregnancy was gained from maternal trabecular and cortical sites and that calcium needed for infant growth during lactation was drawn mainly from the maternal trabecular skeleton in our patients. The effect of pregnancy and lactation on the maternal bone mass was spontaneously compensated after weaning".

In [3], the authors, in their investigation, have "evaluated (19971999) a total of 193 Mexican women, in the first, second and third trimesters of pregnancy, to test the hypothesis that maternal bone lead burden is associated with increasing maternal whole blood and plasma lead levels over the course of pregnancy and that this association is modified by rates of maternal bone resorption.
The results confirm previous evidence that bone resorption increases during pregnancy, with consequential significant release of lead fromda bone, constituting an endogenous source of prenatal exposure".

In [4], it is indicated -1-in pregnancy and bone health, that: (i) "pregnant women absorb calcium from food and supplements better than women who are not pregnant. This is especially true during the last half of pregnancy, when the baby is growing quickly and had the greatest need for calcium"; (ii) "during pregnancy, women produce more estrogen, a hormone that protects bones; (iii) "any bone mass lost during pregnancy is typically restored within several months after the baby's delivery (or several months after breastfeeding has stopped); -2- in breastfeeding and bone health, that: "studies have shown that women often lose 3 to 5 percent of their bone mass during breastfeeding, although they recover it rapidly after weaning. This bone loss may be caused by the growing baby's increased need for calcium, which is drawn from the mother's bones. Women also may lose bone mass during breastfeeding because they are producing less estrogen, which is the hormone that protects bones".

In [5], we have, in our opinion, an excellent revision on "the influence of pregnancy and lactation on maternal bone health" where are considered several topics including each one of them a conclusion. Here, we indicate the title of some of these topics and respective conclusions: (i) - Bone metabolism during pregnancy and lactation - high calcium demand and estrogen deficiency stimulate bone metabolism during pregnancy and lactation; (ii) Pregnancy, lactation and bone - because of a potent correlation between lactation and pregnancy, both are considered as a combined risk factor; (iii) -Parity and bone - several investigations showed logterm supportive effect of parity on bone. For example, it was observed less bone mineral density decrement in multiparous women compared with primiparous; (iv) - Lactation and bone - bone metabolism is higher in lactating mothers with longer period of breastfeeding than that of non-lactating mothers [6]; "it is hypothesized that after discontinuing breast feeding bone resorption returns to normal while bone formation continues [7]; (v) - Pregnancy, lactation and risk of fracture - "bone loss predisposes patients to bone fractures which may cause disabilities, and work loss and imposes high cost to the society [5]. 
In conclusion, we are in agreement with authors [5]:" it seems that pregnancy itself may lead to bone loss but if followed by lactation, it will have a protective effect on bone density while the duration of lactation and parity may modulate its effects. Further investigation on this topic by considering the study limitations, contributory factors and using new safe techniques such as quantative ultrasometry is highly recommended."

Key words: Pregnancy; Breastfeeding; Gynecology; Obstetrics; Bones and Joint

\section{References}

1. Dunne F, Walters B, Marshall T, Health DA (1993) Pregnancy associated osteoporosis. Clinical Endocrinology 1993: 487-490.

2. More C, Bettembuk P, Bhattoa HP, Balogh A (2001) The effects of pregnancy and lactation on bone mineral density. Osteoporos Int 12: 732 .

3. Téllez-Rojo MM, Hernández-Avila M, Lamadric-Figueroa et al (2004) Impact of bone lead and bone resorption on plasma and whole blood lead levels during pregnancy. American Journal of Epidemiology 160: 668-678.

4. Pregnancy, breastfeeding and bone health/NIH Osteoporosis and related bone diseases National Resource Center. WWW.bones.nih.gov

5. Salari P, Abdollahi M (2014) The influence of pregnancy and lactation on maternal bone health: a systematic review. J Family Reprod Health 8: 135-148

6. Holmberg-Marttila D, Leino A, Sievânen H (2003) Bone turnover markers during lactation postpartum amenorrhea and resumption of menses. Osteoporos Int 14: 103-109

7. Sowers M, Eyre D, Hollis B, Randolph JF, Shapiro B Jannaush ML et al (1995) Biochemical markers of bone tumover in lacting and nonlacting potpartum women, J Clin Endocrinol Metab 80: 2210-6. 